\title{
Diagnosis of genital tuberculosis: correlation between polymerase chain reaction positivity and laparoscopic findings
}

\author{
Venkatesh Jnanashree Arpitha*, Channaveeregowda Savitha, Rangaiah Nagarathnamma
}

Department of Obstetrics and Gynaecology, RRMCH, Bangalore, Karnataka, India

Received: 24 July 2016

Accepted: 26 August 2016

\section{*Correspondence:}

Dr. Venkatesh Jnanashree Arpitha,

E-mail: vjarpitha.1186@gmail.com

Copyright: () the author(s), publisher and licensee Medip Academy. This is an open-access article distributed under the terms of the Creative Commons Attribution Non-Commercial License, which permits unrestricted non-commercial use, distribution, and reproduction in any medium, provided the original work is properly cited.

\begin{abstract}
Background: Female genital tuberculosis (GTB) leads to infertility in young women as it involves the fallopian tube and the endometrium. Since it does not produce any dramatic symptoms, disease remains untreated until the female fertility is totally compromised. Diagnostic hystero-laparoscopy is an integral part of management of infertile women. Visual manifestations of pelvic TB are well described, but subtle signs of subclinical disease are not identified nor correlated with laboratory tests. PCR has been described as a rapid, highly sensitive and specific test for detecting DNA of MTB. Hence it's worth studying whether positive PCR test will help make early diagnosis of GTB. This study correlates laparoscopic visual inspection (LVI) findings with endometrial TB-PCR positivity to diagnose GTB.

Methods: A prospective observational study was done in which infertile women who had clinical and HSG findings suggestive of GTB underwent endometrial TB-PCR and hysterolaparoscopy. Among them 69 endometrial TB-PCR positive cases were included in the study. Clinical and hysterolaparoscopy findings in these cases were reviewed.

Results: GTB is the disease of young women and most commonly present with primary infertility (65.2\%). On laparoscopy $60 \%$ of cases showed positive correlation with endometrial TB- PCR and tubal involvement was seen in majority of cases.

Conclusions: Even though PCR is a very valuable tool in diagnosing genital TB, by itself it can neither confirm nor exclude genital TB. The routine application of endometrial TB-PCR assays in addition to clinical and laparoscopic evaluation carries a great potential in improving diagnosis of genital TB.
\end{abstract}

Keywords: Genital tuberculosis, Endometrial TB-PCR, Hysterolaparoscopy

\section{INTRODUCTION}

Tuberculosis (TB), one of the oldest diseases known to affect humans, is caused by bacteria belonging to the Mycobacterium tuberculosis complex. Although tuberculosis usually presents with pulmonary disease, extra-pulmonary tuberculosis (EPTB) is increasingly encountered. ${ }^{1}$ Female genital tuberculosis (GTB) is an uncommon type of EPTB that can lead to infertility in young women as it mostly involves the fallopian tubes (90\%) and the endometrium (50\%).

Genital tuberculosis almost always occurs secondary to pulmonary tuberculosis (commonest) or extrapulmonary tuberculosis such as gastrointestinal tract, kidneys, skeletal system, meninges and miliary tuberculosis. ${ }^{2}$ Primary genital tuberculosis has also been reported in women whose male partners have acute genitourinary tuberculosis by transmission through infected semen. ${ }^{3}$

The spread of tuberculosis from lungs and other sites is normally haematogenous or by lymphatic. Contiguous spread from nearby abdominal organs like intestines or abdominal lymph nodes can cause genital tuberculosis although less common.10-15\% of women suffering from genital tuberculosis is asymptomatic. Symptomatic patients present with menstrual dysfunction (especially oligomenorrhoea or amenorrhoea), primary or secondary infertility, lower abdominal pain, chronic pelvic pain, and/or a pelvic mass. 
The global prevalence of GTB is estimated to be 8 to 10 million cases and the disease is showing a rise in incidence in developing countries due to increasing population and overcrowding. It is increasing in industrialized countries also as a result of its association with HIV infection and increasing immigrant population from under developed countries. Incidence of genital tuberculosis (GTB) in females is found in 0.75 to $1 \%$ of gynecological cases in India. ${ }^{4}$ The disease is responsible for $5 \%$ of all female pelvic infections and occurs in $10 \%$ cases of pulmonary tuberculosis. ${ }^{5}$ Most of the affected cases belong to reproductive age-group. Hence genital tuberculosis is a common cause of infertility in females. The prevalence of GTB in infertile women is reported to vary from $7-15 \%$ in developing countries to $1-2 \%$ in industrialized countries. ${ }^{6}$ Actual incidence of genital tuberculosis may be more because a large proportion of cases go unreported due to low grade symptomatology and lack of sensitive and specific investigations till now.

Since GTB does not produce any dramatic symptoms most of the times disease remains untreated until the female fertility is totally compromised. And the diagnosis of genital TB has profound implications for the asymptomatic women seeking fertility because the multidrug therapy required for more than 6 months is not very promising in terms of pregnancy outcome and the social stigma associated with TB in our country.

Moreover this silent invader of genital tract tends to create diagnostic dilemma because of varied clinical presentation, the diverse results on the imaging, laparoscopy, histopathology and a mixed bag of bacteriological and serological tests, each of which has its limitation in diagnostic sensitivity and specificity. Hence diagnosis of GTB for early institution of treatment remains a clinical challenge.

The gold standard for diagnosis of Tuberculosis is mycobacterial detection and isolation. While direct microscopy in AFB smear and culture in LowensteinJensen medium (LJ) are great success in pulmonary TB, it is of lesser value in genital TB. The reason being these methods have very low sensitivity as in the genital tissue the bacterial load is not much to be detected easily. And bacteria take 4-5 weeks to show growth on LJ media. ${ }^{7-11}$ Nevertheless, even the liquid culture methods such as BACTEC system or more recent introduction of BacTAlert 3D neither reduce the turnaround time below 12 days nor improves the rate of positivity beyond a limit.

The conventional Mantoux test for tuberculin sensitivity is positive in over $40 \%$ of normal healthy individuals in India and has a sensitivity of $55 \%$ and specificity of $80 \%$. Hence Mantoux test is unhelpful in the diagnosis.

Morphological imaging on hysterosalpingography (HSG) and ultrasonography have low sensitivity and specificity for diagnosis of GTB. ${ }^{2,8}$
In the recent years, the molecular diagnostic methods hold the key to the future of the better and efficient diagnosis of genital tuberculosis. Polymerase chain reaction (PCR) has been described as a rapid, highly sensitive and specific molecular biological method for detecting DNA of Mycobacterium tuberculosis in both pulmonary and extra pulmonary samples from suspected tuberculosis patients. ${ }^{9,10,12}$ PCR is an efficient technique with the theoretical possibility of amplifying one DNA sequence of interest to 106 copies in the course of one working day. PCR enables detection of less than $10 \mathrm{pg}$ of DNA that corresponds to about three genome equivalents, thus making it a highly sensitive tool. Endometrial TBPCR assays targets various gene segments, including a 65 $\mathrm{kDa}$ protein-encoding gene (Noel b et al), the IS6110 element (Eisenach et al, Kolk et al) and the mpt 64 gene (Manjunath et al, Seth et al, Dar et al). False PCR positivity due to contamination occurring in clinics and laboratories is one of genuine concern. This can be reduced by strict laboratory design and discipline in collection and processing of specimens, handling of reagents and use of blocking reagents. In case of false negative results, there are individual problems of appropriate sample collection, extraction and assays. False negatives may be caused by inadequate number of organisms and inhibitors in specimen.

Both diagnostic and operative laparoscopy and hysteroscopy are an integral aspect of management of infertile women. Visual manifestations of abdominal and pelvic TB at laparotomy and laparoscopy in established cases are well described. ${ }^{13-18}$ However, subtle signs of subclinical disease have not been identified nor have they correlated with laboratory tests.

Hence because of obvious demand for a reliable and rapid means of diagnosing genital $\mathrm{TB}$ for public health reasons it would be worthwhile to study whether positive PCR test is likely to help in making an early diagnosis and treatment of genital TB. This study describes correlates and compares laparoscopic visual inspection (LVI) findings with endometrial TB-PCR positivity to diagnose GTB.

\section{METHODS}

Cross sectional, prospective observational study was done for one and a half years. Infertile women undergoing hystero-laparoscopy as a part of infertility workup at a tertiary care hospital were taken as study data.

\section{Inclusion criteria}

All infertile women suspected of genital tuberculosis, who undergo hystero-laparoscopy as a part of infertility work up and show positive endometrial TB PCR Result. 


\section{Exclusion criteria}

- Women not giving consent for hystero-laparoscopy.

- Women who show negative endometrial TB-PCR Result.

\section{Method}

- Explanation of procedure to all women participating in the study.

- Consent from every women participating in this study.

- All women had: detailed history, general examination, abdominal examination, local examination, Mantoux test, $\mathrm{CBC}$, pelvic scan, hysterosalpingogram. In relevant cases hysterolaparoscopy was performed in the pre-ovulatory period usually on day 6th to 10th of the cycle and pipelle endometrial sample was taken for TB-PCR.

\section{Endometrial TB-PCR}

Endometrial sample was taken with the help of pipelle's sampling curette. The sample taken is sent for DNA TBPCR. TB-PCR of the endometrial sample was done using the Real time TB-PCR in which there is amplification and detection of the IS6110 gene locus.

\section{Hystero-laparoscopy}

Diagnostic laparoscopy was performed using a $5 \mathrm{~mm} / 10 \mathrm{~mm}$ stryker laparoscope with a 30 degree deflection angle telescope powered with a fibreoptic cable for light source. A careful evaluation of the fallopian tubes, ovaries, pelvic peritoneum, pouch of Douglas and peritoneal cavity was done. Features suggestive of genital tuberculosis were looked for by noting the presence of: suprahepatic adhesions, pelvic adhesions, pelvic congestion, bowel/omental adhesions, peritubal adhesions, cornual block, delayed spillage, fimbrial phimosis, hydrosalphynx, granulomas, short tubes, sacculations/beaded tubes, rigid tubes, TO mass, other incidental findings: fibroids, endometriosis, PCOS.

Following this hysteroscopy using normal saline as the distension media was done to identify features suggestive of tuberculosis that included: cervical stenosis, poor endometrium, bald endometrium, distorted ostia, tubercles, microcaceations, calcifications, and synechiae.

Chromopertubation was done and any delayed or absent spillage of dye was noted. Video recording of all the cases was done. In cases found to be positive for genital tuberculosis by TB PCR, the clinical findings were analyzed and video recordings of the hysterolaparoscopic findings were carefully reviewed for the presence of features suggestive of genital tuberculosis.
Laparoscopic criteria for diagnosis of GTB were grouped as either suggestive (type 1) or non-suggestive (type 2 ) of GTB.

\section{Type I}

\section{Suggestive of GTB}

- Confirmatory: Clear evidence of GTB in the form of multiple granulomas, exudates, loculated fluid, adhesions, hydrosalpinx, Rigid and blocked tubes.

- Suspicious: Subtle signs of chronic inflammation, e.g., patent tubes with pelvic congestion, only a few scattered small granulomas, fimbrial agglutination and phimosis, tubal sacculation, mild adhesions and straw-coloured fluid in of Douglas and peri-hepatic adhesions.

\section{Type II}

Non- suggestive of GTB: Normal findings.

Results of Positive PCR for endometrial TB were then collaborated with hystero-laparoscopic findings.

\section{Statistical methods}

Descriptive and inferential statistical analysis has been carried out in the present study. Results on continuous measurements are presented on mean \pm SD (min-max) and results on categorical measurements are presented in number (\%). Significance is assessed at 5\% level of significance.

The following assumptions on data are made

- Dependent variables should be normally distributed

- Samples drawn from the population should be random, and Cases of the samples should be independent.

\section{Statistical software}

The Statistical software namely SAS 9.2, SPSS 15.0, Stata 10.1, MedCalc 9.0.1 ,Systat 12.0 and R environment ver.2.11.1 were used for the analysis of the data and Microsoft word and Excel have been used to generate graphs, tables etc.

\section{RESULTS}

A prospective observational study was done in which infertile women who had clinical and HSG findings suggestive of GTB underwent endometrial TB-PCR and hysterolaparoscopy for further evaluation. Among them 69 endometrial TB-PCR positive cases were included in the study. Clinical and hysterolaparoscopy findings in these cases were carefully reviewed. 
Table 1: Age distribution.

\begin{tabular}{|lll|}
\hline Age in years & No of patients $(n=69)$ & $\%$ \\
\hline $20-30$ & 43 & 62.3 \\
\hline $31-40$ & 26 & 37.7 \\
\hline
\end{tabular}

Mean age observed in our study was (Mean \pm SD): $29.89 \pm 4.23$ years.

Table 2: Nature of infertility

\begin{tabular}{|lll|}
\hline Nature of IF & No of patients $(n=69)$ & $\%$ \\
\hline Primary infertility & 45 & 65.2 \\
\hline $\begin{array}{l}\text { Secondary } \\
\text { infertility }\end{array}$ & 24 & 34.8 \\
\hline
\end{tabular}

In our study $65.2 \%$ of patients presented with primary infertility and $34.8 \%$ patients presented with secondary infertility. In our study majority of the patients $(76.8 \%)$ had regular menstrual cycle and rest $24.2 \%$ patients presented with menstrual abnormality. Hypomenorrhoea was the most common menstrual abnormality present in $8.7 \%$ patients followed by oligomenorrhoea in $5.8 \%$ of patients, menorrhagia in $5.8 \%$ of patients, amenorrhea in $2.9 \%$ of patients.

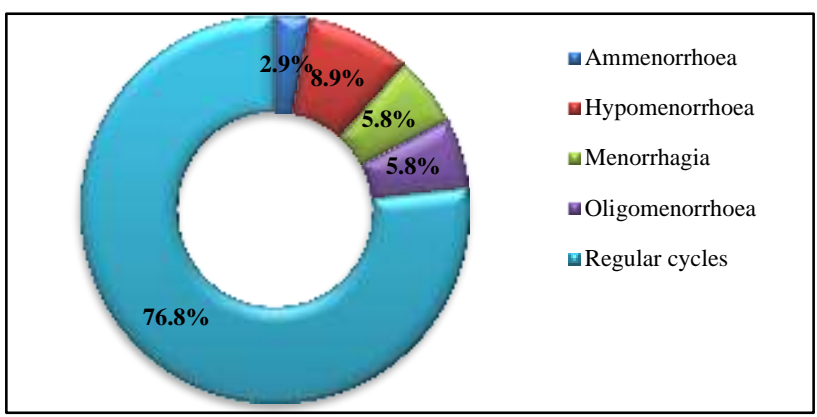

Figure 1: Menstrual pattern.

Table 3: Previous history of PTB/EPTB.

\begin{tabular}{|lll|}
\hline Prev. H/0. of TB & No. of patients $(\mathbf{n = 6 9 )}$ & $\%$ \\
\hline Pulmonary & 4 & 5.9 \\
\hline Genital & 0 & 0.0 \\
\hline Abdomen/intestinal & 2 & 2.8 \\
\hline Bone/joint & 1 & 1.4 \\
\hline Nil & 62 & 89.9 \\
\hline
\end{tabular}

Among the 69 patients in the study, previous history of pulmonary TB was seen in $5.9 \%$ of patients, abdominal TB was seen in $2.8 \%$ of patients, $1.4 \%$ of patients had history of TB of bones. In our study, among 69 cases of positive endometrial TB-PCR none of the cases shown positive results on TB BACTEC (culture) or by $\mathrm{ZN}$ staining.
Indications for doing endometrial TB-PCR and laparoscopy

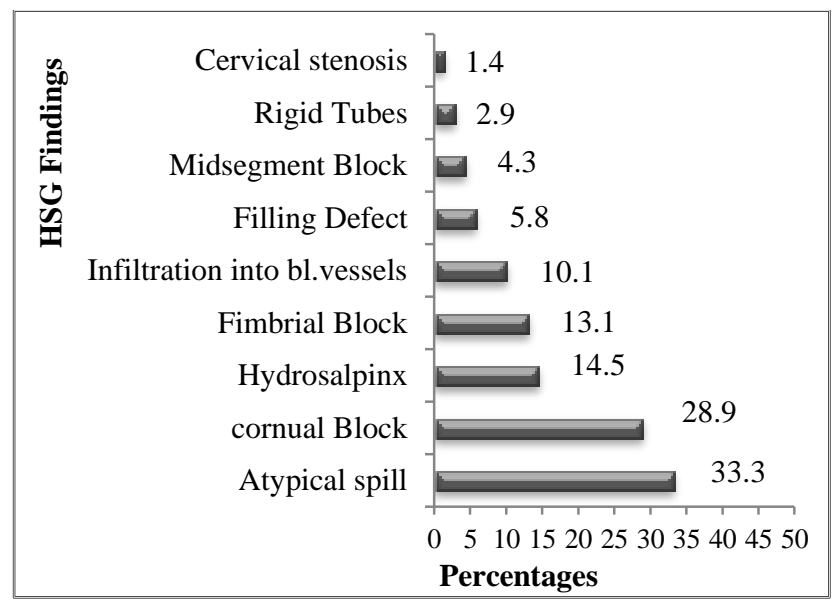

Figure 2: HSG findings.

Table 4: Culture and AFB findings in endometrial TB-PCR positive cases.

\begin{tabular}{|lll|}
\hline Pipelle & No. of patients $(n=69)$ & $\%$ \\
\hline PCR & 69 & 100.0 \\
\hline Culture (bact) & 0 & 0.0 \\
\hline AFB smear & 0 & 0.0 \\
\hline
\end{tabular}

Table 5: Laparoscopic findings in endometrial TBPCR positive cases.

\begin{tabular}{|lll|}
\hline Laparoscopy findings & $\begin{array}{l}\text { No. of patients } \\
(\mathbf{n}=69)\end{array}$ & $\%$ \\
\hline Suprahepatic adhesion & 13 & 18.8 \\
\hline Pelvic adhesion & 24 & 34.8 \\
\hline Pelvic congestion & 4 & 5.8 \\
\hline Bowel/omental adhesions & 11 & 15.9 \\
\hline Peritubal adhesions & 19 & 27.5 \\
\hline Cornual block & 15 & 21.7 \\
\hline Delayed spillage & 18 & 26.0 \\
\hline Fimbrial phimosis & 6 & 8.7 \\
\hline Hydrosalpinx & 11 & 15.9 \\
\hline Granulomas & 3 & 4.3 \\
\hline Shortened tubes & 3 & 4.3 \\
\hline $\begin{array}{l}\text { Sacculations/beaded } \\
\text { tubes }\end{array}$ & 14 & 20.2 \\
\hline Rigid tubes & 3 & 4.3 \\
\hline TO mass & 4 & 5.7 \\
\hline $\begin{array}{l}\text { Other incidental } \\
\text { findings(Endometriosis) }\end{array}$ & 9 & 13.1 \\
\hline
\end{tabular}

In our study $40 \%$ of patients had no evidence of GTB on laparoscopy. In rest $60 \%$ of patient on laparoscopy had: suprahepatic adhesions in 13 patients (18.8\%), pelvic adhesions in 24 patients $(34.8 \%)$, pelvic congestion in 4 patients $(5.8 \%)$, peritubal adhesions in 19 patients $(27.5 \%)$, omental and bowel adhesions in 11 patients 
(15.9\%) and TO-mass in 4 patients $(5.7 \%)$. On chromopertubation, delayed spillage of the dye was seen in 18 patients $(26 \%)$, cornual block in 15 patients (21.7\%), sacculations in 13 patients (20.2\%), hydrosalpinx in 11 patients $(15.9 \%)$, shortened tubes in 3 patients $(4.3 \%)$,rigid tubes in 3 patients $(4.3 \%)$ and fimbrial phimosis in 6 patients $(8.7 \%)$. Other incidental finding was endometrioma (in the form of endometriotic spots) was seen in 9 patients (13.1\%).

Based on the laparoscopic visual inspection (LVI) Findings: 41 patients $(60 \%)$ belong to type 1 (features suggestive of GTB) and 28 patients (40\%) belong to type 2 group (features not suggestive of GTB/normal laparoscopic findings).

Type 1 group was further divided into: Type 1Aconfirmatory findings of GTB (31 patients) and Type 1Bsuspicious findings of GTB (10 patients).

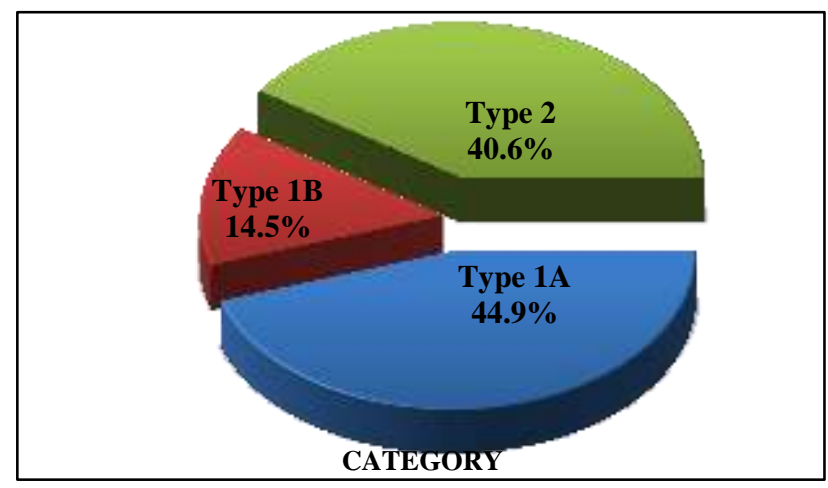

Figure 3: Laparoscopic visual inspection findings.

Table 6: Hysteroscopic findings.

\begin{tabular}{|lll|}
\hline Hysteroscopy & No. of patients $(\mathbf{n = 6 9 )}$ & $\%$ \\
\hline Normal findings & 51 & 73.9 \\
\hline Abnormal findings & 18 & 20.3 \\
\hline Cervical stenosis & 1 & 1.4 \\
\hline Poor endomerium & 8 & 11.6 \\
\hline Bald areas & 4 & 5.8 \\
\hline Distorted Ostia & 2 & 2.9 \\
\hline Synechiae & 1 & 1.4 \\
\hline Fibrosis & 2 & 2.9 \\
\hline Tubercles & 0 & 0.0 \\
\hline Calcification & 0 & 0.0 \\
\hline Others & 4 & 5.8 \\
\hline Endometrial polyp & 2 & 2.9 \\
\hline $\begin{array}{l}\text { Polypoidal } \\
\text { endomerium }\end{array}$ & 2 & 2.9 \\
\hline
\end{tabular}

In our study hysteroscopy revealed normal findings in 51 of patients $(73.9 \%), 2$ patients $(2.9 \%)$ each had distorted ostia and fibrosis, poorly vascularised endometrium was seen in 8 patients $(11.6 \%)$, bald areas was seen in 4 patients $(5.8 \%)$, synechiae was seen in 1 patient $(1.4 \%)$, cervical stenosis in 1 patient $(1.4 \%)$. None of the patients had tubercles or calcification.

\section{DISCUSSION}

With the advent of effective chemotherapy and the wider use of IVF, the outlook on infertile women suffering from GTB has improved significantly. ${ }^{19-23}$ But the vast majorities of patients are not diagnosed in the earlier stages and miss the benefits of early treatment. Hence there is a need for more reliable and rapid diagnostic criteria for early diagnosis of the disease.

A number of reports have described the importance of Endometrial TB-PCR for early diagnosis of GTB and other extra pulmonary TB. ${ }^{9-12,24}$ In India, endometrial TBPCR is also used as screening method for GTB but it demands more evaluation as it is debatable whether PCR positivity indicates infection or disease of the genital tract. By treating all asymptomatic TB-PCR-positive cases, are patients being over-treated? Do the high TBPCR positive rates represent laboratory contamination and false-positivity, or do they confirm the diagnosis of early GTB? Unfortunately, it is generally not possible to substantiate the diagnosis by mycobacterial culture as GTB is a paucibacillary disease. Rigid quality control of PCR is therefore important. In the interests of early treatment, other questions are generally overlooked in favour of a clinical diagnosis of GTB on the basis of TBPCR.

In our study infertile women who had clinical and HSG findings suggestive of GTB underwent endometrial TBPCR and Hysterolaparoscopy for further evaluation. Among them 69 endometrial TB-PCR positive cases were included in the study. Clinical and hysterolaparoscopy findings in these cases were carefully reviewed.

Classically, female genital tuberculosis has been described as a disease of young women, with $80-90 \%$ of patients diagnosed between 20-40 years of age 25 and most of the women present with infertility. In our study the mean age of women with genital tuberculosis was 29 years and $65.2 \%$ patients present with primary infertility and rest $34.8 \%$ patients presented with secondary infertility.

Primary genital tuberculosis is extremely rare. In most series, a history of previous diagnosis of or treatment for extra genital tuberculosis is present in $25-50 \%$ of patients. $^{3,25}$ In our study $7(11.1 \%)$ patients had a previous history of extra genital TB.

The menstrual cycle may be regular and undisturbed in many cases of genital TB. Abnormal uterine bleeding in genital TB has been reported in $10 \%-40 \%$ of patients. ${ }^{26,27}$ In our study majority of the patients had a normal menstrual cycle $(76.8 \%)$ and $24.2 \%$ patients presented with menstrual abnormality. Hypomenorrhoea was the 
most common menstrual abnormality present in $8.7 \%$ patients followed by oligomenorrhoea in $5.8 \%$ of patients. Similar findings were reported in an Indian study where normal menstrual pattern was seen in $57.6 \%$ of patients and most common menstrual abnormality was hypomenorrhoea $(30.1 \%)$ followed by oligomenorrhoea $(3.5 \%){ }^{28}$

In our study, among 69 cases of positive TB-PCR none of the cases shown positive results on TB BACTEC (culture) and by $\mathrm{ZN}$-staining. In few other reports in which mycobacterial cultures were performed, positivity rates were between $10 \%$ and $15 \%$ of GTB patients diagnosed on clinical and morphological criteria and a positive TB-PCR. ${ }^{8-11,29}$ PCR positive and BACTEC (culture) and smear negative cases could be explained by the fact that even though smear and culture has remained a gold standard in diagnosis of pulmonary $\mathrm{TB}$, the very low sensitivity for diagnosis of paucibacillary disease limits its practical utility in extra-pulmonary $\mathrm{TB}^{30-33}$ Hence the use of PCR is suggested as an alternative test especially in culture-negative GTB.

Laparoscopy and hysteroscopy are part of the standardized protocol for evaluation of infertile women. Several reports have described findings suggestive of GTB. ${ }^{8,15,34}$ In our study laparoscopy demonstrated peritubal and periovarian adhesions in $37.5 \%$ cases and omental and bowel adhesions in $15.9 \%$ cases. On chromopertubation, delayed spillage of the dye was seen in $26 \%$ cases, cornual block in $21.7 \%$ cases, sacculations in $20.2 \%$ cases, hydrosalpinx in $15.9 \%$, and fimbrial phimosis in $8.7 \%$ cases. This is due to the fact that fallopian tubes are the initial and most frequently affected site in pelvic mycobacterial infection.

Normal laparoscopic findings were observed in $40 \%$ of positive TB PCR which could be due to the fact that laparoscopy generally detects macroscopic changes that are seen in chronic stages. ${ }^{36}$ This is a very important observation which further emphasizes the fact that genital TB would be more frequently diagnosed if a high index of suspicion is considered in the evaluation of every infertile patient, especially in areas where TB is endemic.

An interesting observation of this study is that $13.1 \%$ of patients along with features suggestive of TB had associated endometriosis evidenced by endometriotic spots. In a study done by Asha B et al, endometriosis was associated with $10.7 \%$ of TB-PCR positive cases. There is no proven theory to justify the association of genital TB with endometriosis but perhaps the change in intrauterine milieu and vascularity might play some role in provoking retrograde spillage of endometrial cells resulting in endometriosis. ${ }^{35}$

In our study hysteroscopy revealed normal findings in 51 cases $(73.9 \%)$ there by implicating less than $50 \%$ endometrial involvement as also been reported by most authors. This can be explained by the fact that unlike the fallopian tubes, the endometrium is comparatively immune to damage by Tubercular bacilli, because of the monthly shedding and repair cycle. Normal looking or non-specific findings of periosteal congestion, etc. can be highly subjective and misleading.

In some of very advanced cases in whom the endometrium was significantly destroyed and adequate endometrial tissue was not available, PCR was often negative. In such cases, EB TB-PCR was nonrepresentative either because of inadequate samples or because this was old, healed disease. TB is well described as a significant underlying etiology in many cases of intrauterine synechiae. $^{37}$

Another finding noted in our study was the presence of cervical stenosis in $1.4 \%$ of cases which could be due to adhesion in the cervical canal. This finding will be of special clinical significance in infertile women. Various studies have reported cervical involvement in $5-10 \%$ of the cases in the form of ulcerative lesions which has a different clinical presentation and implication. ${ }^{9}$

\section{Based on the laparoscopic visual inspection (LVI)}

\section{Findings}

41 patients $(60 \%)$ belong to type 1(features suggestive of GTB) and 28 patients (40\%) belong to type 2 group (features not suggestive of GTB/ normal laparoscopic findings). Type 1 category was further divided into: type 1A- confirmatory findings of GTB (31 patients) and type 1B- suspicious findings of GTB (10 patients).

Hence $60 \%$ cases showed positive correlation with endometrial TB-PCR on laparoscopy which is a significant finding. Implying that endometrial TB-PCR and laparoscopy are complementary tests and the two together can effectively confirm an early clinical diagnosis of GTB.

\section{CONCLUSION}

Genital tuberculosis is the disease of young women and majority of them present with primary infertility.

Majority of the women have an asymptomatic presentation, hence a high degree of suspicion is needed in the evaluation of these patients.

Tubal disease appears to be the most common factor causing infertility in women with GTB.

Since GTB is paucibacillary disease, PCR appears to a rapid and highly sensitive diagnostic modality. But by itself it can neither confirm nor exclude genital TB.

Endometrial TB-PCR and Laparoscopy are complementary tests and together can effectively confirm an early clinical diagnosis of GTB. 
Funding: No funding sources

Conflict of interest: None declared

Ethical approval: The study was approved by the Institutional Ethics Committee

\section{REFERENCES}

1. Chow TW, Lim BK, Vallipuram S. The masquerades of female pelvic tuberculosis: case reports and review of literature on clinical presentations and diagnosis. J Obstet Gynaecol Res. 2002;28:203-10.

2. Rozati R, Agiri SR, Rajeshwari CN. Evaluation of women with infertility and genital tuberculosis. J Obstet Gynecol India. 2006;56:423-6.

3. Sutherland AM, Glean ES, Farlane MJR. Transmission of genitourinary tuberculosis. Health Bull. 1982;40:87-91.

4. Arora R, Rajaram P, Oumachigui A, Arora VK. Prospective analysis of short course chemotherapy in female genital tuberculosis. Int J Gynecol Obstet. 1992;38:311.

5. Varma TR. Genital tuberculosis and subsequent fertility. Int J Gynecol Obstet. 1991;35:1.

6. Parikh FR, Naik N, Nandkarni SG, Soonawala SB, Kamat SA, Parikh RM. Genital tuberculosis: a major pelvic factor causing infertility in Indian women. Fertil Steril. 1997;67:497-500.

7. Abebe M, Lakew M, Kidane D, Lakew Z, Kiros K, Harobe M.Female genital tuberculosis in Ethiopia. Int J Gynecol Obstet. 2004;84:241-6.

8. Jindal UN. An algorithmic approach to female genital tuberculosis causing infertility. Int J Tuberc Lung Dis. 2006;10:1045-50.

9. Bhanu NV, Singh UB, Chakraborty M. Improved diagnostic value of PCR in the diagnosis of female genital tuberculosis leading to infertility. J Med Microbiol. 2005;54:927-31.

10. Baum SE, Dooley DP, Wright J, Kost ER, Storey DF. Diagnosis of culture-negative female genital tract tuberculosis with involvement of polymerase chain reaction. J Reprod Med. 2001;46:929-32.

11. Kumar P, Shah NP, Singhal A. Association of tuberculous endometritis with infertility and other gynaecological complaints of women in India. J Clin Microbiol. 2008;46:4068-70.

12. Roy H, Roy S, Roy S. Use of polymerase chain reaction for diagnosis of endometrial tuberculosis in high-risk subfertile women in an endemic zone. J Obstet Gynecol India. 2003;53:260-3.

13. Sharma JB, Roy KK, Pushpraj M, Kumar S, Malhotra N, Mittal S. Laparoscopic findings in female genital tuberculosis. Arch Gynecol Obstet. 2008;278:359-64.

14. Tinelli A, Malvasi A, Vergara D. Abdominopelvic tuberculosis in gynecology: laparoscopical and new laboratory findings. Aus NZ J Obstet and Gynecol. 2008;48:90-5.

15. Shrama JB, Malhotra M, Arora R. Fitz-Hugh-Curtis syndrome as a result of genital tuberculosis: a report of three cases. Acta Obstet Gynecol Scand. 2003;82:295-7.

16. Muhim AA. Laparoscopic diagnosis of peritoneal tuberculosis. Surg Endosc. 2004;18:1757-61.

17. Volpi E, Calgara M, Ferrero A, Viagno L. Genital and peritoneal tuberculosis: potential role of laparoscopy in diagnosis and management. J Am Assoc Gynecol Laparosc. 2004;11:269-72.

18. Krishna UR, Sheth SS, Motashaw ND. Place of laparoscopy in pelvic infl ammatory disease. J Obstet Gynecol Ind. 1979;29:505-10.

19. Pouillot R, Gerbier G, Gardner IA. 'TAGS', a program for the evaluation of test accuracy in the absence of a gold standard. Prev Vet Med. 2002;53:67-81.

20. Soussis I, Trew G, Matalliotakis I. In vitro fertilization treatment in genital tuberculosis. J Assist Reprod Genet. 1998;15:378-80.

21. Jindal UN, Jindal SK, Dhall GI. Short course chemotherapy for endometrial tuberculosis in infertile women. Int J Gynaecol Obstet. 1990;32:756.

22. Marcus SF, Rizk B, Fountain S, Brinsdon P. Tuberculous infertility and in vitro-fertilization. Am J Obstet Gynecol. 1994;171:1593-6.

23. Gurgan T, Urman B, Yarati H. Results of in-vitro fertilization and embryo transfer in women with infertility due to genital tuberculosis. Fertil Steril. 1996;65:367-370.

24. Gupta N, Sharma JB, Mittal S, Singh N, Misra R, Kukreja M. Genital tuberculosis in Indian infertility patients. Int J Gynecol Obstet. 2007;97:135-8.

25. Schaefer G. Tuberculosis of the genital organ. Am J Obstet Gynecol. 1965;91:714-20.

26. Simon HB, Weinstein AJ, Pasternak MS. Genitourinary tuberculosis: clinical features in a general hospital population. Am J Med. 1977;63:410.

27. Daly JW, Monif GRG: Mycobacteria. In Monif GRG (ed): Infectious diseases in obstetrics and gynaecology. 2nd ed.. Philadelphia, Harper and Row, 1982:301.

28. Sharma JB, Roy KK, Pushparaj M, Kumar S, Malhotra N, Mittal S. Laparoscopic findings in female genital tuberculosis. Arch Gynecol Obstet. 2008;278(4):359-64.

29. Abebe M, Lakew M, Kidane D, Lakew Z, Kiros K, Harobe M. Female genital tuberculosis in Ethiopia. Int J Gynecol Obstet. 2004;84:241-6.

30. Katoch VM. Newer diagnostic techniques for tuberculosis. Ind J Med Res. 2004;120:418-28.

31. Tripathy SN, Triapthy SN. Laparoscopic observation of pelvic organs in pulmonary tuberculosis. Int $\mathrm{J}$ Gynecol Obstet. 1990;32:129-31.

32. Ramachandaran R, Paramasivan $\mathrm{CN}$. What is new in the diagnosis of tuberculosis? ICMR Bulletin. 2002;32:69-76.

33. Dye C, Watt C, Bleed DM, Hosseini SM, Raviglione MC. Evolution of tuberculosis control and prospects for reducing tuberculosis incidence, prevalence and deaths globally. J Am Med Asso. 2005;293:2790-3. 
34. Volpi E, Calgara M, Ferrero A, Viagno L. Genital and peritoneal tuberculosis: Potential role of laparoscopy in diagnosis and management. J Am Assoc Gynecol Laparosc. 2004;11:269-72.

35. Asha B, Hansali N, Manila K, Priti S, Dhawal B. Genital tuberculosis in infertile women: Assessment of endometrial TB PCR results with laparoscopic and hysteroscopic features. Journal Obstetrics Gynecology. 2011:301-6.

36. Oosthuizen AP, Wessels PH, Hefer JN. Tuberculosis of female genital tract in patients attending an infertility clinic. South Aft Med J. 1990;77:562-4.
37. Sharma JB, Roy KK, Pushpraj M. Genital tuberculosis: an important cause of Asherman's syndrome in India. Arch Gynecol Obstet. 2008;277:37-41.

38. Jindal UN, Bala Y, Sodhi S, Verma S, Jindal S. Female genital tuberculosis: early diagnosis by laparoscopy and endometrial polymerase chain reaction. Int J Tuberc Lung Dis. 2010;14(12):162934.

Cite this article as: Arpitha VJ, Savitha C, Nagarathnamma R. Diagnosis of genital tuberculosis: correlation between polymerase chain reaction positivity and laparoscopic findings. Int J Reprod Contracept Obstet Gynecol 2016;5:3425-32. 